\title{
Thermography for Complex Regional Pain Syndrome
}

Konkuk University Medical Center, Konkuk University School of Medicine

Anesthesiology and Pain Medicine

Jae Hun Kim

Complex regional pain syndrome (CRPS) is one of the most severe pain diseases of neuropathic pain. Complex regional pain syndrome is frequently an intractable and challenging disease for both patients and physicians. Its severe pain and chronic course can result in significant disability for the patient. There is no single pathognomonic test for diagnosis of CRPS.

Patients with CRPS not only develop severe pain but also exhibit multiple symptoms such as allodynia, hyperalgesia, temperature asymmetry, skin color change, edema, sweating change, motor dysfunction, and trophic change.[1,2]

There is no single pathognomonic test for diagnosis of CRPS. Patients with CRPS can have variable and multiple symptoms.[3] Some patients with other types of neuropathic pain can have also have the severe pain and some of the other symptoms of CRPS. The diagnosis of CRPS is based on both patients' symptoms and on objective diagnostic signs. Therefore, accurate diagnosis of CRPS is challenging for physicians.

In the fall of 2003, a closed workshop was held in Budapest, Hungary.[4] The Budapest concensus group approved and codified empirically derived criteria as a revision of the IASP diagnostic criteria. The IASP committee on taxonomy recently approved and codified these so-called Budapest Criteria as 'the new IASP criteria' (Table 1).[5]

The clinical diagnostic criteria resulted in a sensitivity of 0.85 and a specificity of 0.69 , which represented a good compromise in identifying as many patients as possible at an acceptably accurate rate in the clinical context.[5] The research diagnostic criteria resulted in a sensitivity of 0.70 and a specificity of 0.94 . Of all those tested, this decision rule resulted in the highest probability of accurate diagnosis for both CRPS and non-CRPS patients (approximately 80\% and 90\% accuracy, respectively), even when a relatively low occurrence rate for 
CRPS was assumed.[5]

To diagnose CRPS using clinical diagnostic criteria, a patient must report at least three of four symptom categories, and a physician must confirm at least two of four sign categories. Similarly, using research diagnostic criteria, a patient must report all four-symptom categories, with physician confirmation of at least two of four sign categories. Physicians usually carry out several special tests to objectively detect the positive signs of the diagnostic criteria. One of the special tests is thermography. Some of the special tests can evoke severe pain in CRPS patients. However, thermography can detect the different temperature of CRPS patient's pain site without evoked pain. There are several investigations about the temperature differences for diagnosis of CRPS. Recently, over $1^{\circ} \mathrm{C}$ of temperature difference is usually accepted for diagnosis of CRPS.

Table 1. Revised CRPS criteria by the Budapest consensus group [4,5]

\section{General Features of the Syndrome}

CRPS is a syndrome characterized by a continuing (spontaneous and/or evoked) regional pain that is seemingly disproportionate in time or degree to the usual course of any known trauma or other lesion. The pain is regional (not in a specific nerve territory or dermatome) and usually has a distal predominance of abnormal sensory, motor, sudomotor, vasomotor, and/or trophic findings. The syndrome shows variable progression over time.

There are two versions of the proposed diagnostic criteria: a clinical version meant to maximize diagnostic sensitivity with adequate specificity, and a research version meant to more equally balance optimal sensitivity and specificity.

\section{Clinical diagnostic criteria for complex regional pain syndrome}

1) Continuing pain, which is disproportionate to any inciting event

2) Must report at least one symptom in three of the four following categories

Sensory: Reports of hyperalgesia and/or allodynia

Vasomotor: Reports of temperature asymmetry and/or skin color changes and/or skin color asymmetry Sudomotor/Edema: Reports of edema and/or sweating changes and/or sweating asymmetry

Motor/Trophic: Reports of decreased range of motion and/or motor dysfunction (weakness, tremor, dystonia) and/or trophic changes (hair, nail, skin) 
3) Must display at least one sign* at time of evaluation in two or more of the following categories

Sensory: Evidence of hyperalgesia (to pinprick) and/or allodynia (to light touch and/or deep somatic pressure and/or joint movement)

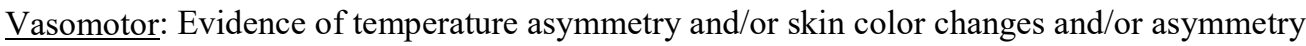

Sudomotor/Edema: Evidence of edema and/or sweating changes and/or sweating asymmetry

Motor/Trophic: Evidence of decreased range of motion and/or motor dysfunction (weakness, tremor, dystonia) and/or trophic changes (hair, nail, skin)

4) There is no other diagnosis that better explains the signs and symptoms

For research diagnostic criteria, must report at least one symptom in each of the four categories and must display at least one sign* at time of evaluation in two or more of the categories

* A sign is counted only if it is observed at time of diagnosis

References

1. Harden RN. A clinical approach to complex regional pain syndrome. Clin J Pain 2000; 16: S26-32.

2. Rockett M. Diagnosis, mechanisms and treatment of complex regional pain syndrome. Curr Opin Anaesthesiol 2014; 27: 494-500.

3. Wasner G, Schattschneider J, Binder A, Baron R. Complex regional pain syndrome-diagnostic, mechanisms, CNS involvement and therapy. Spinal Cord 2003; 41: 61-75.

4. Harden RN, Bruehl S, Stanton-Hicks M, Wilson PR. Proposed new diagnostic criteria for complex regional pain syndrome. Pain Med 2007; 8: 326-31.

5. Harden RN, Oaklander AL, Burton AW, Perez RS, Richardson K, Swan M, et al. Complex regional pain syndrome: practical diagnostic and treatment guidelines, 4th edition. Pain Med 2013; 14: 180-229. 
http://dx.doi.org/10.21611/qirt.2017.013 